\title{
SIGNIFICADOS ATRIBUIDOS A LA EDUCACIÓN : PERCEPCIONES DE NIÑOS Y NIÑAS DEL SERVICIO NACIONAL DE MENORES SENAME CHILE
}

\author{
MEANINGS ATTRIBUTED TO EDUCATION: PERCEPTIONS OF CHILDREN UNDER \\ NATIONAL SERVICE SENAME CHILE
}

\section{Mónica Hernández Z. ${ }^{1}$, Daniel Lagos C. ${ }^{2}$, Sandra Mella D. ${ }^{3}$}

\begin{abstract}
Resumen
La educación formal constituye un pilar fundamental para los procesos de integración social que viven los niños, niñas y adolescentes que han sido vulnerados en sus derechos. Sin embargo, las experiencias escolares negativas que han vivido, podrían generar opiniones contrarias en relación al papel que cumple la educación formal en sus vidas. El objetivo de la presente investigación se orienta a conocer y describir cuál es el sentido, propósito y significado que tiene la educación formal para los niños y niñas que residen en el Centro de Reparación Especializado de Administración Directa (CREAD Galvarino), dependiente del Servicio Nacional de Menores de Chile (SENAME).

La investigación fue de tipo cualitativa con un enfoque fenomenológico, y la recolección de datos se realizó mediante la aplicación de una entrevista semi-estructurada y un cuento proyectivo que es considerado como un método que aprecia la subjetividad en un sentido global u holista. El análisis de datos implicó la codificación de categorías analíticas clasificadas y agrupadas en las dimensiones de sentido, propósito y significado.

En los resultados, se observa que experiencias escolares positivas agregan valor a ésta ocupación, que el propósito puede ser a corto o a largo plazo, y que el significado está notablemente influenciado por las opiniones que el entorno vierte respecto de la educación formal. Además, se encontraron diferencias significativas en las respuestas dadas por los niños que asistían a la Escuela " $A$ " y la Escuela " $B$ ".

Los resultados promueven la reflexión respecto del rol que deben cumplir los CREAD en relación al derecho a la educación y a la resignificación de la educación formal en aquellas personas que están vivenciando un proceso de restitución de derechos e integración social.
\end{abstract}

\section{Palabras Claves:}

Educación formal, ocupación, sentido, propósito, significado, integración social

1 Terapeuta Ocupacional Licenciada en Ciencias de la Ocupación Humana, CRS Cordillera Oriente - Unidad Medicina Física y Rehabilitaciónlsabelhz.to@gmail.com

2 Terapeuta Ocupacional Licenciado en Ciencias de la Ocupación Humana, Oficina de Discapacidad de Maipú, Municipalidad de Maipú, Centro de Salud Mental COSAM Cerrillosdlagosceron@gmail.com

3 Terapeuta Ocupacional, Magíster en Integración de Personas con Discapacidad, Licenciada en Ciencia de la Ocupación, Profesor Asistente Universidad de Chile. Escuela de Terapia Ocupacional ,smella@med.uchile.cl 


\begin{abstract}
Formal education constitutes a main support to the process of social integration of children and adolescents with rights violations. However, the negative experiences they had lived in educational sphere might generate contrary opinions related to role that formal education have in their lives. The objective of this investigation is oriented to know and describe the sense, purpose, and meaning that formal education has for children living in a Center of Direct Administration Repair CREAD, under the National Youth Service of Chile (SENAME). .

The research was qualitative with a phenomenological approach and data collection was performed by applying a semi-structured interview and a projective story is considered as a method to appreciate subjectivity in a global or holistic sense. Data analysis involved coding analytical categories sorted and grouped the dimensions of meaning, purpose and meaning.

The results show that positive scholar experiences adds value to this occupation, that purpose can be short-term or long-termed, and that the meaning is markedly influenced by the opinions social environment do about formal education. Also, significant differences were found between answers given by children that attended "School A" and "School B".

Results promote reflection regarding to the role CREAD must accomplish related to right to education, and about the redefinition of formal education meaning in those persons that are experiencing a process of rights restoration and social integration.
\end{abstract}

\title{
Key words:
}

Formal education, occupation, sense, purpose, meaning, social integration.

\section{INTRODUCCIÓN}

El Servicio Nacional de Menores SENAME en Chile es un organismo gubernamental que depende del Ministerio de Justicia, al cual son derivados niños y niñas infractores de ley y/o vulnerados de derechos. Cuando el Estado chileno ratificó la Convención Internacional de los Derechos del Niño (Instituto de estudios avanzados USACH, 2005), el SENAME se convirtió en el instrumento primordial de la implementación de una política de protección de derechos, establecida en la Ley 16.618 destinada a los niños y niñas, y sus familias en circunstancias de riesgo y vulnerabilidad social (Gobierno de Chile, 2012). De ahí se desprende el Área de Protección de derecho, que complementando la acción del Estado, se ha configurado para la intervención tres niveles, de acuerdo a la complejidad (leve, mediana, alta) de las situaciones que afectan a ésta población.

Los niños y niñas que presentan las situaciones de mayor complejidad, son atendidos en el tercero de los niveles establecidos por el área de Protección de derecho, el cual se orienta a la reparación de problemas de alta severidad, tales como: maltrato grave, explotación sexual comercial, niños/as en situación de calle, consumo habitual de drogas, explotación laboral de niños y adolescentes, etc. Para esta población, la oferta de protección considera las siguientes modalidades: Residencias especializada, programas complementarios con foco en la familias (Fortalecimiento Familiar, Familias de Acogida), Centros de Intervención Especializada (PIE) destinados a una población que reúne varios problemas y debe ser atendida en conformidad a su complejidad, y los Programas Especializados de Administración Directa.

Los Centros de Reparación Especializados de Administración Directa CREAD, corresponden a una de las modalidades incluidas en la oferta programática del Área de Protección de derecho, y brindan atención a la población mayor de seis años, gravemente vulnerada en sus derechos, que presenta situaciones de alta complejidad y que requiere recibir atención bajo la modalidad residencial. El objetivo general de los CREAD corresponde a: "Contribuir a la restitución de derechos y la re-significación de experiencias de vulneración presentes en la población infanto-adolescente en situaciones de alta complejidad y que requieran de una medida residencial por disposición de Tribunales de Familia". Mientras que su propósito se define como: "Reparar el daño que presentan los niños, niñas y adolescentes, menores de 18 años derivados por juez competente del Tribunal de Familia, que cuentan con una medida de protección por ser expuestos a experiencias crónicas de vulneración de derechos" (SENAME, 2013). El proceso de intervención se inicia con el ingreso CREAD junto a un proceso continuo de intervención, focalizado en el respeto a sus derechos y la reparación de estos en consideración a su propia diversidad. Esta modalidad de intervención se centra en la re-significación de sus propios estilos de relación en un clima de respeto y valoración, entregando así una oportunidad a estos de una rutina diaria estructurada, protegida y en un espacio de acogida. 
El área educativa es una de las principales a trabajar para fortalecer a este grupo objetivo y dar las herramientas necesarias para desenvolverse autónomamente en la sociedad. La educación es un derecho estratégico, un derecho que abre las puertas al goce de los demás derechos, una puerta de entrada indispensable a los procesos de integración (Crotti, 2004). La evaluación realizada a cada niño y niña, deberá entregar lineamientos claros a aquellos encargados del ámbito educacional, quienes en conjunto con profesionales psicólogo/trabajador social definirán bajo qué modalidad estará inscrito el niño para continuar sus estudios o bien para regularizarlos, en caso de existir un retraso escolar de acuerdo a lo esperado en el rango etario en el que se encuentra (SENAME, 2013). Sobre este último punto es necesario que todos los intervinientes tengan en consideración cómo se encuentra el niño para enfrentar la asistencia a escuela formal, en términos de no sentirse discriminado, incapaz de rendir según lo esperado, víctima de burlas por pares, entre otros.

En el caso de los niños/as y adolescentes que han ingresado a la red SENAME por situaciones de vulneración de sus derechos, la participación en la educación formal se ha caracterizado por ser un proceso intermitente y con escasos resultados positivos (Dávila, Ghiardo, Hatibovich, \& Oyarzún, 2008). En general se trata de niños, niñas y adolescentes que están insertos en el sistema escolar, principalmente en establecimientos de educación municipal. En algunos casos se asume cierto nivel de desajuste y retraso en las trayectorias educativas, fundamentalmente como consecuencia de la repitencia y la deserción; sin embargo, estos problemas parecieran no relacionarse tanto con el acceso y la permanencia, que están más o menos resueltos, sino con los códigos de las escuelas y del proceso educativo mismo. Se habla de relaciones conflictivas con la escuela, de desacatos a la autoridad, relaciones problemáticas con los pares, problemas de aprendizaje y de déficit en los desempeños escolares. Esta caracterización resulta preocupante, si pensamos que la educación corresponde a uno de los pilares fundamentales que sustentan la integración social de ésta población.

La integración social desde una óptica o enfoque sicosocial incorpora no sólo los planos de oportunidad y acceso a la estructura económico-social, sino que también considera el intercambio que realiza el individuo con su entorno simbólico y cultural (Instituto de estudios avanzados, USACH, 2005). La integración social se constituye en la relación que hay entre subjetividad y estructura social en diferentes ámbitos, en cuanto a dimensiones macro y micro-sociales, en un cierto contexto histórico/cultural, en el cual se pone en juego la posibilidad de pertenencia a un todo social (Espinoza, 2004). En los niños y niñas que han sido vulnerados en sus derechos, la experiencia en la educación formal no se ha configurado de la misma forma que en la población promedio, originándose diferencias tanto por las situaciones de vida, como por las experiencias escolares que han tenido, y dando cabida a interpretaciones particulares de ésta actividad. Surge entonces la siguiente interrogante:

\section{¿Qué interpretación hacen los niños y niñas vulnerados en sus derechos, respecto de la educación formal?}

Desde una perspectiva ocupacional, entenderemos la educación formal como la ocupación principal del área de desempeño educativa. En palabras de la AOTA, la educación formal comprende las categorías de academia (por ej. matemáticas, lectura, estudiar para obtener un título), no académico (recreos, colaciones, traslados), actividad extracurricular (deportes, bandas, bailes), y participación vocacional (AOTA, 2008). Situados en el contexto de nuestra investigación realizada en el CREAD Galvarino, entenderemos la educación formal como aquella ocupación que comprende las actividades académicas (asistir a clases) y no académicas (recreos, traslados) realizadas dentro de la escuela y fuera de ella (realizar tareas y estudiar), pero no incluiremos las actividades extracurriculares, puesto que no es un ítem presente en la vida cotidiana de nuestra población de estudio.

\section{Elementos subjetivos de la Educación Formal como una ocupación.}

Diversos autores han aportado definiciones de lo que ellos consideran como ocupación, encontrándose en ellas elementos que convergen en la idea de que la ocupación humana se compone de una dimensión altamente subjetiva. Los científicos ocupacionales al igual que los terapeutas ocupacionales comparten el interés por entender el significado subjetivo de las ocupaciones, es decir, el propósito, sentido y pertinencia que tienen para la persona que las lleva a cabo.

La tendencia actual en la ciencia ocupacional en el mundo es a conceptualizar las ocupaciones de acuerdo 
a cuatro criterios: por su forma o rasgos observables, por sus atributos intrínsecos que benefician al individuo y al contexto, por los significados y apreciaciones que construyen los sujetos durante el proceso ocupacional o por los propósitos humanos que cumplen (Grupo de Investigación Ocupación y Realización humana, 2011). Estas conceptualizaciones han permitido abordar el concepto desde diversas perspectivas, muchas de ellas complementarias.

Las ocupaciones pueden entenderse como actividades de vida diaria, nombradas, organizadas y a las que el individuo y una cultura le dan valor y significado (Larson, Wood y Clark, 2005).Ocupación es cada cosa que las personas hacen para ocuparse a sí mismas, incluyendo el cuidado de sí mismos... gozar de la vida... y contribuir al entretejido social y económico de sus comunidades (Law, Polatajko, Baptiste, \& Townsend, 1997 ). Otra forma, es comprender a la ocupación como una relación dinámica entre una forma ocupacional, una persona con una estructura de desarrollo única, significados y propósito subjetivo, y el desempeño ocupacional resultante (Nelson \& Jepson-Thomas, 2003). Gómez S. por su parte, establece que si al realizar cualquier tipo de actividad se tiene un propósito y un significado, se transforma en ocupación (Gómez, 2001). También existen enfoques que la relacionan con la producción de identidades personales desarrolladas a partir de ellas (Christiansen, 1999) o como estructuras con funciones diversas como evolutivas y sociales entre otras (Wilcock, 2006).

De estas y otras definiciones se extraen al menos tres elementos subjetivos que conforman la construcción que el sujeto elabora de una ocupación: el sentido, el propósito y el significado. Iwama señala que el significado de un acontecimiento o de la ocupación variará según las experiencias y los contextos compartidos únicos que lo conforman (Iwama, 2008), es por esto que los significados que les atribuimos a nuestras actividades son únicos y pueden apreciarse a través del relato de la cosmología y las visiones del mundo determinadas en cada contexto.

El sentido es el "valor" personal o individual que se da a las actividades, el que se construye desde las experiencias sensoriales, afectivas, motoras, cognitivas, sociales, espirituales, que se acumulan a lo largo de la vida (Comité de Ciencias de la Ocupación Humana ETOUCH, 2007). El propósito corresponde a lo que se quiere conseguir con una ocupación, anticipación del resultado de lo que vamos a realizar (Gómez, 2001). Y el significado corresponde al contenido simbólico colectivo presente en una ocupación, que no necesariamente abarca a la interpretación subjetiva que tiene lugar en la persona cuando realiza una ocupación. Es entendido a través del proceso de culturización, el que es mediado principalmente por el lenguaje. Es importante mencionar que pese al relevante papel que juegan estos elementos subjetivos en los procesos de integración social, existe escasa literatura desde Terapia Ocupacional y desde el campo Educativo, referente a investigaciones o análisis teóricos que exploren o incorporen estos elementos.

Reuniendo los aportes de los autores ya mencionados, en el marco de esta investigación una ocupación será entendida como: "aquella actividad con sentido y propósito en la que la persona participa cotidianamente y que adquiere significado a través de los aportes de la cultura", considerándola de esta forma como un concepto que triangula estas tres perspectivas. Esta nueva definición, nos permitirá indagar en los elementos subjetivos que construyen a la educación formal como una ocupación, y que están presentes en niños/as residentes en un CREAD. De esta forma la pregunta de investigación a responder será:

\section{¿Cuál es el sentido, propósito y significado que tiene la educación formal para los niños y niñas que residen en el CREAD Galvarino?}

El objetivo de la investigación, por tanto, es describir el sentido, propósito y significado que tiene la educación formal para los niños y niñas del CREAD Galvarino, que han sido vulnerados en sus derechos y que están vivenciando un proceso de integración social.

\section{Caracterización del contexto educativo de CREAD Galvarino}

Actualmente, el CREAD Galvarino ha logrado matricular al $70 \%$ de su población en establecimientos educacionales, el $13 \%$ se encuentra en proceso de diagnóstico y evaluación, el $11 \%$ está inscrito para rendir exámenes libres, y el $6 \%$ está en espera de obtener una matrícula.

CREAD Galvarino, ubicado en la comuna de Estación Central, está vinculado a cinco establecimientos educaciones cercanos a la residencia, tres de ellos corresponden a educación regular: Escuela" $A$ ", Escuela "B" y Escuela "C", donde asiste el 43\%, 23\% y $2 \%$ de 
los niño escolarizados, respectivamente. Los otros dos centros son escuelas especiales: Escuela "D" y Escuela " $E$ ", a la primera asiste el $14 \%$ de los niños escolarizado, y a la segunda asiste el 18\% (Estadísticas Departamento Educación CREAD Galvarino, 2013)

Los profesionales de CREAD Galvarino han establecido relaciones informales con los establecimientos educacionales que matriculan a sus niños y niñas, por ende, el acceso a continuidad o nivelación educativa queda en manos de la capacidad de gestión por parte de los profesionales. Además, declaran que lo ideal sería contar con un convenio con uno o más establecimientos educacionales, puesto que permitiría una matrícula segura una vez que el niño lo requiera (cualquier fecha del año), adicionalmente no sería necesario depender de relaciones informales para que los niños ingresen al recinto educacional, sino que exista un compromiso de éste para con los estudiantes y CREAD. De este modo, se podría cumplir con la orden del Tribunal, que indica que todos los niños deben ser escolarizados una vez que ingresan al CREAD, excepto en las siguientes situaciones:

- Casos de connotación pública (posible seguimiento público y de fiscalía).

- Niños y niñas con tratamiento psiquiátrico Niños y niñas que hacen abandono del CREAD.

En ciertas ocasiones, aunque el Tribunal exija que se cumpla la orden de escolarización, el CREAD Galvarino no puede dar respuesta por los siguientes motivos:

- La dupla psicosocial evalúa que no es pertinente el ingreso del niño a un establecimiento educacional, ya que prima su tratamiento de salud mental, por ejemplo, siendo derivado a Hospital de Día para niños y adolescentes.

Falta de vacantes en establecimientos educacionales, lo que se traduce en una larga espera.

Como respuesta educativa para aquellos niños que no son escolarizados, se implementó dentro de CREAD Galvarino, un Programa Escolar Interno (PEI) que consiste en un modelo de enseñanza multrigrado del MINEDUC, es decir, los niños y niñas que ingresan a este programa, a pesar de tener variadas diferencias entre sí, como los años de retraso académico y edad, tienen en común no haber podido acceder a educación formal.

Las Escuelas Especiales " $D$ " y " $E$ " reciben a aquellos niños y niñas que presentan trastornos conductuales o continuo abandono escolar, además de sufrir algún tipo de discapacidad. Cabe destacar que por la falta de vacantes en centros educativos, algunos niños sin discapacidad son matriculados en estas escuelas especiales.

La Escuela "A" se destaca por recibir niños con mayores problemas conductuales y retraso escolar, entregando una atención más personalizada, ya que cada curso cuenta con 7 u 8 alumnos. No se exige uso de uniforme institucional, basta con el uso de buzo. Además, tiene una jornada más breve que la mayoría de los colegios. Es un recinto con limitada infraestructura, muy pequeño y precario

La Escuela "B" se caracteriza por recibir niños sin retraso escolar, exige el uso de uniforme y tiene horarios establecidos sin ningún tipo de flexibilidad; por estas razones, los profesionales de CREAD Galvarino, deciden matricular en este reciento a aquellos niños y niñas que tengan un historial de buen rendimiento académico previo, así evitan la frustración por la alta exigencia del establecimiento.

\section{MetodoloGíA}

La investigación es cualitativa de tipo fenomenológico, ya que se centra en una experiencia vivida por el sujeto, impregnada de su subjetividad. Según este enfoque, se pretende describir y entender un fenómeno desde el punto de vista de cada participante y desde la perspectiva construida socialmente (Hernández, Fernández, \& Baptista, 2006). En esta investigación, se indaga sobre el fenómeno de la educación formal para los niños y niñas de CREAD Galvarino, ubicado en la comuna de Estación Central, definiendo el significado, sentido y propósito que tiene ésta ocupación para cada sujeto.

\section{Muestra}

En éste contexto y para obtener una muestra lo más representativa posible, los criterios de selección de los sujetos fueron: 
- Niños/as vulnerados en sus derechos y que residan en CREAD Galvarino.

- Niños/as que asistan al colegio y en jornada de la tarde.

- Niños/as entre 8-12 años.

Los criterios de exclusión se ajustan a los niños que no cumplen con los requisitos anteriormente señalados.
En total, se entrevistó a 16 sujetos, de ellos 8 eran hombres y 8 mujeres, fueron seleccionados aleatoriamente. Del total de niñoas/as, 8 niños/as asisten a "Escuela Saint Henry School" (Letra A). , 6 niños/as pertenecen a "Escuela Básica Provincia de Chiloé" (Letra B). y 2 niños/as forman parte de la Escuela Especial "Nuestra Señora del Buen Consejo" (Letra D). Ver Tabla 1. Antecedentes Generales de Niños y Niñas del estudio.

Tabla 1. Antecedentes Generales de Niños y Niñas del estudio

\begin{tabular}{|c|c|c|c|}
\hline Nombre & Género & Edad & Escuela a la que asiste \\
\hline F.D. & Masculino & 10 & D \\
\hline M.H. & Masculino & 9 & B \\
\hline T.C. & Masculino & 12 & $\mathrm{~A}$ \\
\hline D.D. & Masculino & 10 & $\mathrm{~A}$ \\
\hline C.V. & Femenino & 12 & $\mathrm{~B}$ \\
\hline C.E & Femenino & 8 & $\mathrm{~A}$ \\
\hline N.M. & Femenino & 12 & $\mathrm{~A}$ \\
\hline K.A. & Masculino & 10 & $\mathrm{D}$ \\
\hline G.S. & Femenino & 11 & $\mathrm{~B}$ \\
\hline D.L. & Masculino & 12 & $\mathrm{~A}$ \\
\hline A.H. & Masculino & 9 & $\mathrm{~A}$ \\
\hline S.M. & Masculino & 11 & $\mathrm{~B}$ \\
\hline R.T. & Femenino & 10 & $\mathrm{~B}$ \\
\hline B.S. & Femenino & 12 & $\mathrm{~A}$ \\
\hline P.Z. & Femenino & 8 & $\mathrm{~B}$ \\
\hline G.P. & Femenino & & \\
\hline
\end{tabular}

\section{ReCOlECCIÓN DE DATOS}

La información fue recabada por medio de dos instrumentos. El primero corresponde a una entrevista semi-estructurada, donde cada pregunta estaba asociada a uno de los componentes de la ocupación a investigar: propósito, sentido y significado (Anexo 1). El segundo es un cuento de tipo proyectivo, cuya historia era leída a los entrevistados y correspondía a una situación muy familiar a la que ellos vivían en ése momento. El cuento cumple con la finalidad de confirmar o complementar la información obtenida en la entrevista, puesto que la técnicas proyectivas son considerada como un método que aprecia la subjetividad en un sentido global u holista (Sneiderman, 2011). El test proyectivo no evalúa la subjetividad en el sentido cuantitativo puro, sino que forma parte de un proceso de toma de decisiones que parte de un modelo teórico o conceptual, en este caso el sentido, propósito y significado de la ocupación de estudiar, e intenta comprender y analizar un fenómeno 
concreto, que puede ser real o simbólico y que puede darse en un aspecto manifiesto y/o latente.

El proceso de acercamiento y recolección de datos, incluyó reuniones con los profesionales de CREAD Galvarino, visitas de observación a los niños/as dentro de los espacios del hogar, y las entrevistas a cada niño y niña.

Se realizó una invitación a participar y la entrega de un consentimiento informado a cada uno de los entrevistados y a cada una de las duplas psicosociales a cargo, exponiendo objetivos y características de la investigación, beneficios y formas de entregar los datos. En este proceso se precisó sobre la confidencialidad de los datos y el uso investigativo de esta.

El protocolo de consentimiento fue reviso por los investigadores y expertos en el tema, aplicando previamente pruebas que permitieron asegurar la comprensión de los aspectos comunicados en este.

El proceso participativo en la investigación es de carácter voluntario. Se obtuvo el total de los consentimientos firmados.

\section{Resultados}

Los resultados de la investigación fueron obtenidos a partir del análisis cualitativo de contenidos de las entrevistas realizadas y del test proyectivo. El análisis implicó la identificación de patrones reiterados en las respuestas dadas por los entrevistados, que posteriormente fueron agrupados y clasificados en diversas categorías de análisis. En un primer momento se obtuvieron 12 categorías analíticas que conforman los principales hallazgos de nuestra investigación, y fueron divididas de acuerdo a las dimensiones de sentido, propósito y significado ya definidas en el instrumento de recolección de datos. Luego, a partir de éstos resultados, se decidió realizar un análisis comparativo de las respuestas obtenidas entre los dos principales colegios incluidos en la muestra.

1. Sentido. El sentido de la educación formal está constituido por la relación entre la motivación, autoeficacia, disfrute e importancia que los entrevistados otorgan a ésta ocupación y que se conforman a partir de sus experiencias. A partir del análisis de las respuestas, se obtienen seis categorías analíticas:

Categoría analítica 1: Existen elementos que motivan la participación de los niños y niñas en la educa- ción formal. Las principales motivaciones que indican los entrevistados para participar de la educación formal corresponden a poder aprender más, hacer tareas y participar de las asignaturas preferidas por cada uno. En menor medida, puesto que sólo se menciona por algunos, se reconocen los espacios recreativos y la participación social como otros motivadores presentes. Destaca el hecho de que dos niños indican que otra de sus motivaciones para asistir al colegio es al aumento de su sensación de libertad. En esa categoría analítica, los niños responden a las preguntas: " $i$ Te gusta ir al $\mathrm{CO}^{-}$ legio? ¿Por qué?/ ¿Qué cosas te gustan del colegio?"

Para la mayoría de los niños, aumentar el conocimiento, hacer tareas y las asignaturas preferidas, motivan la participación. Lo anterior se evidencia con la siguiente cita:

Niña, 10 años: "Sí, porque aprendo más, del cuerpo humano, a sumar, a restar, a dividir.

Me gusta todo, ir al recreo, estar en la sala, hacer tareas, porque lo paso bien"

En cuanto a otros factores motivantes, aparecen las relaciones sociales y la recreación, como compartir con compañeros de colegio durante el recreo, jugando o socializando. A continuación, se presenta una cita a modo de ejemplo:

$$
\begin{aligned}
& \text { Niño, } 11 \text { años: "Sí (...) porque me gusta } \\
& \text { salir al patio, me gusta jugar, me gusta } \\
& \text { compartir" }
\end{aligned}
$$

Otro factor que motiva la participación, es aquel que se relaciona con pasar más tiempo fuera del CREAD, lo cual es relacionado por los niños con sensación de libertad, como se expresa en esta respuesta:

Niño, 10 años: Me gusta ir porque me siento más... libre para salir de aquí y también me gusta ir porque nos enseñan y tenemos más amigos también"

Niña, 11 años: "Me gusta ir al colegio 
pa`estar con mis compañeros más que nada, y pa` estar un poco más libre. Entrevistador: ¿A qué te refieres con estar un poco más libre?

Niño: Es que es por estar encerrado acá"

Categoría analítica 2: Existen elementos que desmotivan la participación de los niños y niñas en la educación formal. Así como se refieren elementos que motivan la participación en la educación formal, también se mencionan razones que disminuyen la motivación.

Una de las principales razones que emergen para no querer asistir al colegio es la baja motivación por asignaturas que consideran difíciles o aburridas, así se expresa en la siguiente respuesta:

Niña, 10 años: "Entrevistador ¿Qué cosas son las que menos te gustan del colegio?

Niño: Las menos que me gustan serían matemáticas...

Entrevistador: ¿Por qué?

Niño: Porque me cuesta un poco más"

Además, cuando existe una percepción negativa del desempeño docente, los niños y niñas desmotivan su participación como estudiantes, pues perciben poco compromiso de parte de los profesores.

Niño, 12 años: "Entrevistador: ¿Por qué no te gustan los profes?

Niño: No porque son más o menos pencas

Entrevistador: ¿Por qué?

Niño: pencas, porque hacen la tarea, y después... chao, pa' la casa, pa'fuera"
Entre las respuestas de los niños y niñas, se mencionó de manera reiterada el conflicto entre pares que se da dentro del establecimiento educacional, las débiles relaciones interpersonales, e incluso, situaciones de bullying. Algunos niños y niñas, mencionan sentirse segregados de los demás compañeros de escuela por pertenecer a SENAME, razón por la cual comienzan a sentirse distintos al resto que no vive en hogar. Una de las niñas entrevistadas, da cuenta de lo planteado anteriormente:

Niña, 12 años: "Entrevistador: ¿Cómo lo pasas yendo al colegio?

Niño: Más o menos, porque es aburrido. Me siento incómoda allá. Acá no, allá sí.

Entrevistador: Porque la gente, las otras personas, son distintas a mí, siento que allá me diferencian de otras personas. Yo lo noto en la forma de cómo me miran.

Entrevistador: Además de cómo te miran, ¿qué otra cosa has notado?

Niña: Que igual tratan mal a veces, los de quinto."

Los niños y niñas, perciben la infraestructura como un factor importante al momento de sentirse motivados para asistir al colegio, en muchos casos no cuentan con salas, patio y materiales de calidad, por lo tanto, ven mermada su motivación. La siguiente respuesta identifica a gran parte de la población entrevistada:

Niño 9 años "Entrevistador: ¿Qué cosas te gustan del colegio?

Niño: Nada 
Entrevistador: ¿Por qué?

Niño: porque es muy chico el colegio, no se puede hacer nada"

Categoría analítica 3: La autoeficacia percibida por los niños y niñas está relacionada con la opinión que tienen respecto de su propio desempeño conductual y académico. Se evidencia un predominio de los aspectos conductuales al momento de referirse sobre su propio desempeño. Sumado a lo anterior, la tendencia muestra una alta autoeficacia. Las siguientes citas responden a la pregunta "¿Cómo te va en el colegio?":

Niña, 10 años: "Bien, porque cuando hago las tareas la tía me abraza fuerte, porque las tías no me retan, a veces me saco buenas notas"

Niña, 9 años: "Niño: Mal

Entrevistador: ¿Por qué te va mal?

Niño: Eh... más o menos, es que a veces me porto mal"

Categoría analítica 4: Los niños y niñas disfrutan asistiendo al colegio, otro aspecto que configura el sentido que tiene para ellos la educación formal. Ésta categoría muestra una correlación con la categoría 1, debido a que los principales motivadores de la participación permiten a su vez disfrutar de la actividad. En esta categoría, los niños y niñas responden a la pregunta “¿Cómo lo pasas en el colegio?".

En primer lugar, podemos inferior que los niños y niñas disfrutan de las instancias de participación social y recreación, refieren constantemente que lo pasan bien jugando, contando chistes y compartiendo con los demás. En segundo lugar, los niños y niñas disfrutan de las libertades que ellos encuentran en la educación formal, las cuales muchas veces se condicen con situaciones de consumo, como en el siguiente entrevistado:
Niño, 12 años: "Entrevistador: ¿Cómo lo pasas en el colegio?

Niño: Sí...bien (ríe mientras lo dice)

Entrevistador: La pasas bien. ¿Por qué?

Niño: ... en el colegio fumo cigarro y marihuana

Entrevistador: ¿por eso lo pasas bien?

N: Sí. Con amigos más grandes sí...de 14

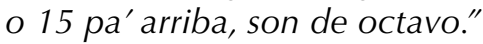

Categoría analítica 5: Los niños y niñas no disfrutan asistiendo al colegio, debido a conflictos entre pares, bullying, condiciones de infraestructura, entre otros. Emerge una relación directa entre aquellos elementos que no motivan la participación en la ocupación, y a su vez no permiten disfrutar de ésta. Los niños y niñas responden a la pregunta "¿Cómo lo pasas en el colegio?".

Niña, 9 años: "A veces aburrido, otras veces bien...me aburro porque el colegio es muy chico, entonces no podemos correr, entonces tenemos que andar caminando."

Niño, 10 años:"Bien, ah no, no, la pasó mal, porque un niñito que se llama C.R. y B.B. me pegan.

Entrevistador: ¿Y tú qué puedes hacer con eso, los acusas?

Niño: No, porque me amenazan."

Categoría analítica 6: La educación formal es importante para los niños y niñas del CREAD Galvarino. Todos los entrevistados concuerdan en que la educación formal ("ir al colegio") corresponde a una ocupación importante en sus vidas, sin embargo, las razones que 
justifican esa importancia son muy diversas. Sólo una de las entrevistadas menciona que la educación formal le va a permitir integrarse de manera adecuada en la sociedad. En esta categoría los niños y niñas responden a la pregunta "¿Qué significa para ti ir al colegio?/¿Es importante para ti ir al colegio?".

En primera instancia, podemos concluir que la importancia de la educación formal radica en pasar de curso, demostrando temor frente a la situación de repitencia. Para otros, la importancia está en aumentar el conocimiento, o bien, utilizar este conocimiento para alcanzar metas futuras, como trabajar. Lo anterior, se pone evidencia con la siguiente cita:

Niña, 11 años: "Entrevistador: ¿Tú crees que es importante ir al colegio?

Niña: Sí... Porque así aprende más, porque para cuando salga puede trabajar

Entrevistador: Ah, cuando salgas poder trabajar ¿Y si no estudias?

Niña: No puedo trabajar"

Otro grupo de niños y niñas, responde que para ellos la importancia de la educación reside en el desarrollo personal, para lograr la integración social, y por lo mismo, evitar situaciones de calle o consumo problemático, por ejemplo.

Niño, 11 años: "Entrevistador: ¿Es importante ir al colegio?

Niño: Sí, para ser alguien en la vida

Entrevistador: ¿Qué pasa si no vamos al colegio?

Niño: No somos nadie en la vida, estaríamos tirados en la calle, drogados, podríamos ser alcohólicos. No quiero eso en mi vida, por eso voy al colegio."
Los hallazgos indican que existen elementos motivantes y desmotivantes en la educación formal, la mayoría de los entrevistados disfrutan de ésta actividad, sin embargo, hay que poner énfasis en aquellos factores que son percibidos de manera negativa, como por ejemplo, el conflicto entre pares. La importancia de participar en ésta ocupación, radica principalmente en poder pasar de curso y aumentar el conocimiento. La percepción de autoeficacia está determinada, en mayor medida, por el desempeño conductual que por el rendimiento académico.

\section{Propósito}

Con respecto a ésta dimensión se pueden observar dos tipos de respuesta. El primer tipo da cuenta de la existencia de un propósito, y que guarda relación con aprovechar las instancias que brinda la educación formal para lograr metas, divertirse o establecer vínculos. Tales propósitos pueden ser a corto o largo plazo, y este último concuerda con una proyección a futuro. Otro tipo de respuestas, evidencian la carencia de un propósito claro, al mencionar que sólo se va al colegio para aprender o para hacer tareas.

Categoría analítica 7: La educación formal tiene un propósito para los niños y niñas.

Un primer grupo, se caracteriza por reconocer un propósito ligado a aumentar su conocimiento y, de esta manera, alcanzar metas. Mientras que otro grupo de niños y niñas, demuestra que su propósito al asistir al colegio, se relaciona con el encuentro de un espació de recreación y/o participación social. Entre las respuestas más representativas a la pregunta "¿Para qué te sirve ir al colegio?", encontramos:

Niña, 10 años:" Para sacar mi carrera, tener mis estudios y todo eso, para ser alguien en la vida"

Niño, 10 años: "Para estudiar y para aprender, más, sobre lo que no sabe."

Niña, 9 años: "Hacer tareas, eeeh... Un poquito, a respirar el aire. Para salir al patio. Me sirve... para compartir." 
Categoría analítica 8: Participar de la educación formal no tiene un propósito a futuro. En una minoría de los casos y contrario a lo descrito en la categoría anterior, en otras respuestas se repite la idea de que solo se va al colegio para estudiar o para aprender, existiendo poca claridad con respecto a la importancia que tiene el conocimiento y el aprendizaje en nuestra sociedad actual. Para ejemplificar ésta situación, podemos destacar las siguientes citas:

Niña, 9 años: "Para aprender

Entrevistador: ¿Por qué es importante aprender?

Niña: Mmmm no sé"

Niño, 9 años "A estudiar...a aprender.

Entrevistador: ¿Para qué te sirve ir al colegio? ¿Por qué?

Niño: No sé, solo voy"

\section{Significado}

Entendemos que el significado alude a la presencia de un contenido simbólico colectivo que no necesariamente abarca la interpretación subjetiva que tiene lugar en la persona cuando se ocupa. No obstante, con respecto a esta dimensión, se concluye que el significado que tiene la educación formal para los niños y niñas se manifiesta en la forma de una opinión propia, influenciada al menos por el significado que el entorno más próximo le otorga a ésta actividad, es decir, los profesores, la familia y el CREAD Galvarino.

Categoría de análisis 9: Los niños y niñas presentan una opinión propia respecto de la educación formal, que ha sido influenciada por el contexto, y que configura el significado personal. Se concluye que los niños y niñas presentan diferentes opiniones respecto del papel que cumple la educación formal en nuestra cultura. Esto se debe a la influencia que ejercen los factores contextuales en las opiniones que cada uno elabora sobre los fenómenos que ocurren. En palabras de Mo- linas de Rondina, siempre se significa en un contexto determinado, en una cultura determinada, en una épo$\mathrm{ca}$, siguiendo pautas que posicionan la experiencia en los discursos culturalmente disponibles. Las construcciones grupales y sociales, como los mitos y creencias colectivas, sostienen y co-construyen significados (Molinas de Rondina, 2006). Esta categoría se desprende de las respuestas dadas ante la pregunta ¿Por qué crees que todas las personas tienen que ir al colegio?

En un primer grupo se observa la ausencia de un significado respecto de la educación formal, que podría deberse a una dificultad para reconocer las razones socio-culturales que fundamentan la existencia de ésta ocupación.

Niño, 10 años: "No sé, ni idea, ni me interesa tampoco."

Niña, 9 años: "No sé, no sé porque todas las personas tienen que ir al colegio"

Por otra parte, hay quienes consideran que la educación formal significa una herramienta de desarrollo personal o de ascenso social, como se aprecia en las siguientes respuestas:

Niña, 11 años: "Para que sean alguien en la vida. Porque estarían tiradas en la calle sin nada en los bolsillos...si vas al colegio tienes tu casa, tu auto, tu esposo, tu perro, todo"

Niño, 10 años: "Porque así aprenden y hacen puras cosas buenas en la vida... portándose bien, haciéndole caso a las tías, a los tíos, y eso"

Niña, 12 años: "Porque es importante, y hay que llegar a cuarto medio, y si nosotros no llegamos a cuarto medio, no podemos trabajar en lo que nosotros queramos. A mí me gustaría ser doctora"

Para otro grupo, participar de la educación formal significa una obligación impuesta por el medio y no se tiene mayor conciencia de los propósitos o utilidades que ésta tiene para el futuro. 
Niña, 10 años: "Porque, como se llama, es obligación ir al colegio para los papás..."

Niño, 11 años: "No sé, y creo que están obligados."

Por último, es importante mencionar que para algunos la educación formal significa un medio que permite evitar una potencial situación de calle. Ésta consideración debe estar muy ligada a las experiencias tempranas que han recibido estos niños y niñas, y a la reproducción del lenguaje utilizado en su cotidianeidad.

Niña, 11 años: "Para que aprendan y no anden en la calle y tengan una vida por delante."

Niño, 9 años: "Si no fueran al colegio los hijos estarían..., no sabrían nada de matemáticanidenada, yestaríanenlacalle."

Categoría de Análisis 10: La familia influye en el significado que los niños y niñas le otorgan a la educación formal. Si consideramos que la cultura influye en los significados, la familia comprende a uno de los elementos más determinantes del contexto con el cual se han relacionados los niños y niñas. Para obtener ésta información, los entrevistados respondieron a la pregunta "¿Qué opinan tus familiares sobre ir al colegio?/i Qué te dicen en tu casa sobre ir al colegio?".

En mayor medida, la familia significa la educación formal como una obligación y enfatiza en el cumplimiento de deberes. En los siguientes ejemplos se observa una opinión centrada en el presente de los niños y niñas, que apela a fomentar el adecuado desempeño y comportamiento en el aula.

Niño, 11 años: "Lo que me dicen que no sea flojo, que no vaya a calentar el asiento y que estudie no más."

Niña, 10 años: "Mi mamá dice que tengo que pasar de curso, que tengo que hacer las tareas, porque si no las hago no puedo pasar de curso, dice que no puedo quedar repitiendo... me dice eso porque ella me quiere y no quiere que quede repitiendo, pero si quedo repitiendo no me reta...pero a veces me reta un poco, pero no tanto."

En otros casos, se deduce que la familia significa la educación formal como algo positivo, pero no se refieren ni se detallan las razones por las cuales se considera así.

Niña, 11 años: "Me dice que igual es fome que no nos puede ir a buscar como lo hacía, pero que es bueno que estemos estudiando porque no nos farreamos el año. Ella me anima a ir al colegio. A veces digo "no quiero ir al colegio" y ella me dice "vai a tener que ir o yo te voy a dejarte de las orejas".

Niña, 10 años: "Que está bien, que ¿¿cómo te fue en el colegio?' y 'ieres buena en el colegio?'..."

En menor medida, se observa que la familia significa la educación formal como una herramienta de desarroIlo personal o ascenso social, como vemos a continuación:

Niña, 9 años: "Que vaya al colegio,

Entrevistador: Y ¿por qué?

Niña: Porque tengo que aprender, para que cuando sea grande tengo que ser una profesora."

Niña, 10 años: "Que aprenda muchas cosas, que me vaya bien, y que sea alguien en la vida...Cuando grande me gustaría ser tía de hogar."

Finalmente, la familia también significa la educación formal como un medio para evitar una potencial situación de calle, o por otro lado, no ha logrado insertar ningún significado al respecto. 
Niño, 9 años: "Que me hace bien a mí, porque no voy a ser un vagabundo cuando grande."

Niño, 11 años: "No se...

Entrevistador: ¿Te han dicho algo?

Niño: no sé realmente

Entrevistador: ¿Algún otro familiar que te haya dicho algo sobre ir al colegio?

Niño: No, nadie"

Categoría de análisis 11: Los profesores influyen en el significado que los niños y niñas le otorgan a la educación formal. Otro de los factores contextuales considerados por los investigadores, y que fue incorporado en los instrumentos de recolección de datos, corresponde a la influencia que ejercen los profesores en las opiniones que elaboran los estudiantes. Las siguientes citas responden a la pregunta "¿Qué le dirían los profesores al niño?", refiriéndose al personaje principal del cuento, quien decide retirarse de la educación formal.

En la mayoría de las respuestas entregadas, los profesores significan la educación formal como una obligación y enfatizan en la asistencia y continuidad. Los niños y niñas refieren que un profesor reaccionaría de la siguiente manera ante la situación descrita:

Niña, 8 años: "Que va a quedar repitiendo por inasistencia, así que no falte."

Niña 11 años: "Que venga a la escuela, porque si no viene a la escuela... no importa que se saque malas notas, lo que importa es que vaya y nunca falte."

Niño, 11 años: "Que le ponga harto empeño, que tiene que seguir intentando."
Por otra parte, también destaca el hecho de que los profesores significan la educación formal como una forma de aumentar el conocimiento, así como se muestra en las siguientes citas:

Niño, 10 años: "Que vuelva al colegio.

Entrevistador: ¿Por qué?

Niño: Porque aprenda más"

Niña, 9 años "Que está mal porque tiene que aprender"

Niño, 11 años "Que siguiera como está ahora, porque va bien en ese camino de aprender."

Sin embargo, los entrevistados no refieren que el profesor signifique la educación formal como una herramienta de desarrollo personal o de ascenso social.

Categoría de análisis 12: El CREAD influye en el significado que los niños y niñas le otorgan a la educación formal. Por último, se considera importante poder obtener los discursos presentes en el CREAD que también crea significados al respecto. Las siguientes citas responden a la pregunta "¿Qué le dirían las personas del hogar al niño?", refiriéndose al personaje principal del cuento, quien decide retirarse de la educación formal.

Al igual que como ocurre con los profesores el CREAD significaría, en mayor medida, la educación formal como una obligación y enfatiza en la asistencia y continuidad.

Niño, 9 años "Que fuera no más al colegio

Entrevistador:¿le darían alguna explicación?

Niño: Porque tiene prueba" 
Nuevamente, se repite también el hecho de que el CREAD significa la educación formal como una obligación, pero a éste factor se le agrega que reprueba la inasistencia.

Niña, 10 años "Me imagino que lo retarían. No, no retar. Que le llamarían la atención porque faltó."

Niño, 10 años: "Que lo castigan por no ir al colegio

Entrevistador: ¿Y por qué lo castigan?

Niño: Porque... porque, porque el colegio, es algo muy especial para la persona

Entrevistador: ¿Y cómo lo castigarían?

Niño: Sin recreo, o dejándolo en la sala o sino.. Haciéndole una copia y haciéndole tareas"

Niño, 12 años: "En el hogar al niño le dirían que nosotros gastamos mucha plata en matricularlo para que él aprenda y que tiene que ir de lunes a viernes."

Finalmente, y en menor medida se refiere que el CREAD significa la educación formal como una forma de aumentar el conocimiento, tal como se aprecia en los siguientes ejemplos:

Niño, 10 años: Anda al colegio

Entrevistador: ¿y por qué le dirían eso?

Niño: porque tiene que aprender po."

Niña, 11 años "Que vaya a la escuela, porque si no va a la escuela, a Javier no le van a dar más estudios, si no sigue yendo a la escuela porque él no quiere ir, y si no quiere ir no va a aprender nada."

\section{Diferencias entre establecimientos educacionales: Escuela "A" y Escuela "B"}

La Escuela "A" se destaca por recibir niños con mayores problemas conductuales y retraso escolar, entregando una atención más personalizada, ya que cada curso cuenta con 7 u 8 alumnos. No se exige uso de uniforme institucional, basta con el uso de buzo. Además, tiene una jornada más breve que la mayoría de los colegios. Es un recinto con limitada infraestructura, muy pequeño y precario

La Escuela "B" se caracteriza por recibir niños sin retraso escolar, exige el uso de uniforme y tiene horarios establecidos sin ningún tipo de flexibilidad; por estas razones, los profesionales de CREAD Galvarino, deciden matricular en este reciento a aquellos niños y niñas que tengan un historial de buen rendimiento académico previo, así evitan la frustración por la alta exigencia del establecimiento.

Sentido. El sentido está determinado, entre otros aspectos, por las experiencias que cada niño y niña ha vivido, para esta investigación el foco está puesto en el fenómeno de la educación formal, obteniendo respuestas que evidencian experiencias negativas y positivas en torno a la escuela. Al indagar en los aspectos negativos de la población que asiste a la Escuela "A", los niños/s son enfáticos en mencionar la precaria infraestructura, horarios de salida muy tardíos, profesores prejuiciosos y poco preocupados, bajas calificaciones, bullying y conflicto entre pares, e inexistencia de motivación para hacer las tareas. En la Escuela "B", las experiencias negativas se centran principalmente en situaciones de buIlying y asignaturas de alta exigencia para su nivel. A lo que se refiere a las experiencias positivas de asistir a la educación formal para los niños/as de la Escuela " $\mathrm{A}$ ", refieren: instancia de evasión al hogar y de disfrute, adquisición de conocimientos y participación social. Para la Escuela "B", se obtienen como experiencias positivas: disposición amable y comprometida de sus profesores, buen rendimiento en asignaturas, desarrollo personal, participación social, buen comportamiento y rendimiento, instancia de adquisición de conocimientos y alta motivación para hacer tareas. 
Propósito. Esta dimensión, corresponde a lo que se quiere conseguir con la educación formal, es decir, una anticipación del resultado que se obtiene al educarse. Los estudiantes que asisten a la Escuela " $\mathrm{A}$ " se limitan a respuestas acotadas al conocimiento y desarrollo personal a futuro, e incluso existe ausencia de propósito. En cambio, aquellos que asisten a la Escuela "B", se caracterizan por expresar que su propósito de la educación es un conjunto de acciones, tanto para realizar en el ahora como en el futuro. Entre las respuestas más reiteradas en este segundo grupo, se encuentran las subcategorías relacionadas con: aprender, estudiar, hacer las tareas, pasarlo bien, relacionarse con otros niños, pasar de curso, ser alguien en la vida, llegar a la universidad. Es decir, tanto el conocimiento, desarrollo personal (presente y futuro), la participación social y el ascenso social, son las áreas ligadas con el propósito.

Significado. Los niños de la Escuela " $A$ " y aquellos de la Escuela " $B$ " presentan similitudes principalmente en el significado que la familia les ha dado sobre la educación, sin embargo, al comparar el significado personal, se evidencia ausencia solamente en el grupo de "A". Además, los niños de este establecimiento, reconocen que el significado de asistir a educación formal, está ligado a la evasión de una potencial situación de calle. Por otro lado, las respuestas de los niños de la Escuela "B" que fueron categorizadas en desarrollo personal, la mayoría corresponde a una proyección futura. Con respecto al significado dado por los profesores, los niños de la Escuela " $\mathrm{A}$ " reiteran de manera constante que los docentes castigan y reprueban la conducta de inasistencia, mientras que los niños del otro establecimiento educacional, refieren que los profesores insisten en la educación como una obligación y adquisición de conocimientos, y en la continuidad de estudios. Finalmente, el significado que da el CREAD Galvarino a la educación, es percibido principalmente como asistencia al centro educacional, para los niños de la Escuela " $A$ "; en cambio, los niños de Escuela "B" mencionan que CREAD Galvarino alude a la educación como un medio de adquirir conocimiento. Ambos grupos concuerdan que CREAD Galvarino significa la educación formal como una obligación que puede ser reprobada y castigada en caso de no cumplir con la asistencia.

\section{DISCUSIÓN}

La presente investigación obtiene resultados diversos y profundos, que permiten confirmar la existencia de elementos subjetivos implicados en la ocupación, descritos y definidos anteriormente por diversos autores. Se logra describir cómo las distintas experiencias determinan el sentido que la educación formal tiene para cada uno de los niños, y cuáles son las razones que subyacen al valor que cada uno le entrega a ésta ocupación. Aspectos como percepción de la infraestructura, las relaciones sociales, las vivencias, el rendimiento, son elementos que van configurando un sentido positivo o negativo de ésta ocupación, y sin duda también repercuten en los propósitos y significados que los niños van elaborando. Se mencionan propósitos de la educación formal a largo o a corto plazo, sin embargo se encuentran diferencias significativas en las respuestas obtenidas entre aquellos que asisten a la Escuela "A" y la Escuela "B". Por otro lado, con respecto al significado que los entrevistados le dan a la educación formal, se concluye la familia, el CREAD y los profesores conforman los elementos culturales más influyentes y/o determinantes en la opinión que el niño(a) expresa sobre ésta ocupación.

El objetivo de esta investigación se logra a partir de la sistematización y vinculación de los datos en las tres grandes dimensiones de análisis ocupacional: el sentido, propósito y significado. Los resultados obtenidos refuerzan la idea de que el proceso ocupacional se realiza y se reconstruye en un entramado subjetivo e indagar en estos aspectos, desde un paradigma construccionista, permite acercarnos de manera más precisa a la realidad de las personas (Flores, 2004). Persson, Erlandsson, Eklund e Iwarsson (2001) también incursionan en las vivencias ocupacionales y refieren que "comprender el significado percibido por la persona es esencial para clarificar la verdadera naturaleza de una ocupación".

La metodología cualitativa presente en esta investigación permitió obtener información personal, ligada a las creencias, valores y experiencias que cada niño guarda. Conocer y describir los elementos subjetivos 
que la educación formal tiene para los niños y niñas del CREAD Galvarino, promueve la reflexión en torno a los propósitos que se propone un CREAD y la factibilidad de lograrlos. ¿Cuál es la contribución a la restitución del derecho a la educación por parte de los CREAD? ¿Existe una re-significación real de las experiencias educativas de vulneración presentes en la población infanto-adolescente? ¿Cómo se re-significa una ocupación que carece de sentido si se repiten las experiencias negativas?

Aumentar la conciencia respecto de la utilidad que tiene la educación formal para estos niños, no sirve si no se otorga la oportunidad tangible de re-significar ésta experiencia. La transformación individual y social radica en utilizar la ocupación de manera consciente para procesos de crecimiento personal e interacción personal y para procesos emancipatorios de equidad y justicia (Townsend, 1997). Olvidar las condiciones educativas en las que se encuentran los niños y niñas que residen en un CREAD constituye un riesgo que podría limitar su desarrollo personal o peor aún, impedir la integración social efectiva.

Este trabajo de investigación permite visualizar áreas de investigación e intervención para la Terapia Ocupacional, y otras disciplinas de las Ciencias Sociales interesadas en los procesos de integración social de una de las poblaciones más vulnerables en nuestro país. La propuesta terapéutica se verá así como la posibilidad de deconstruir y reconstruir la subjetividad mediante ocupaciones creadoras de significado, donde se acompaña a un sujeto protagonista, que tiene competencias, creencias, valores y compromisos propios, que posee habilidades y fortalezas (Molinas de Rondina, 2006). Estas personas, instituciones, y/o comunidades necesitan ampliar sus perspectivas, potenciar los recursos que ya poseen, y para ello también se requiere instalar el debate respecto de cómo asegurar el acceso equitativo a aquellas personas que se encuentran en situación de vulnerabilidad social.

\section{ReferenCias Bibliográficas}

American Occupational Therapy Association (AOTA). (2010). Marco de Trabajo para la Práctica de

la Terapia Ocupacional: Dominio y Proceso, $2^{\circ}$ Edición. Recuperado el 8 de noviembre de 2012 de http://www.terapia-ocupacional. com/aota2010esp.pdf.
Comité de Ciencia de la Ocupación de la Escuela de Terapia Ocupacional de la Universidad de Chile.

(2007). Definición y desarrollo del concepto de Ocupación: ensayo sobre la experiencia de construcción teórica desde una identidad local. Revista Chilena de Terapia Ocupacional, (7), 76-82. Recuperado el 22 de abril de 2013 de http://www.revistaterapiaocupacional.uchile.cl/index.php/RTO/article/viewFile/81/62

Christiansen, Ch. (1999). Defining Lives: Occupation as Identity: An Essay on Competence, Coherence, and the Creation of Meaning. En: The American Journal of Occupational Therapy. 53 (6). Noviembre/Diciembre.

Dávila, O; Ghiardo, F; Hatibovich, \& Oyarzún, A. (2008) ¿Enfoque de Derechos o Enfoque de

Necesidades?. Recuperado el 3 de abril de 2013 de http://www.sename.cl/wsename/otros/2012/ENFOQUE.pdf.

Flores M. (2004). Implicancias de los paradigmas de investigación en la práctica educativa. Revista

Digital Universitaria, 5(1), 2-9. Recuperado el 4 de noviembre de 2012 de http://www.revista.unam.mx/vol.5/num1/art1/ene_art1.pdf.

Gobierno de Chile. (2000). Marco Legal Protección de la Infancia. Recuperado el 29 de agosto de 2013 de http://www.sename. $\mathrm{cl} /$ wsename/estructuras.php?name $=$ Content $\&$ pa $=$ showpage $\& p$ $\mathrm{id}=11$.

Gómez S. (2009). La ocupación y su significado como factor influyente de la identidad personal.

Revista Chilena de Terapia Ocupacional, (3), 43-47. Recuperado el 24 de mayo de 2013 de http://www.revistas.uchile.cl/index.php/ RTO/article/viewArticle/149/131

Grupo de Investigación Ocupación y Realización humana (2011). Ocupación: sentido, realización y

libertad. Diálogos ocupacionales en torno al sujeto, la sociedad y el medio ambiente. Recuperado el 20 de abril de 2013 de http:// www.bdigital.unal.edu.co/5794/7/9789587197297.pdf

Hammell, K. (2004), Dimension of Meaning in the Occupations of Daily Life. Canadial Journal of Occupational Therapy. 71(5), 296-305. Recuperado el 4 de octubre de 2013 de http:// www.caot.ca/CJOT_pdfs/CJOT71/Hammell71(5)296_305.pdf

Instituto de estudios avanzados de la Universidad de Santiago (2005). Efectos de los Programas del

SENAME en la Integración Social de los Niños y Niñas. Recuperado el 20 de marzo de 2013 de http://www.sename.cl/wsename/otros/ doc_sename/Efectos_prog_Sename.pdf. 
Iwama, M.(2008). "En Busca de una Terapia Ocupacional Culturalmente Relevante". Revista Terapia Ocupacional Galicia. 5(2), 1-28. Recuperado el 2 de septiembre de 2013 de http:// www.revistatog.com/num8/pdfs/maestros.pdf.

Larson, E.; Wood, W.; \& Clark, F. (2005). Ciencia ocupacional: desarrollo de la ciencia y la práctica de la ocupación a través de una disciplina académica (pp. 15-26). En: E. B. Crepeau, Cohn, E.S., \& B.A Boyt Schell, (eds.). Terapia Ocupacional. Décima edición. Bogotá: Editorial Médica Panamericana.

Mena P., (s.f.) La Educación no formal y su aporte al trabajo con niños-as y familias en situación de pobreza. Recuperado el 14 de abril de 2013 de http://www. superacionpobreza.cl/EditorFiles/File/ ADOPTA $\% 20$ UN\%20HERMANO/Documentos\%20adopta/educacionnoformal.pdf.

Persson D, Erlandsson L-K, Eklund M, Iwarsson S. (2001). Value dimensions, meaning, and

complexity in human occupation-a tentative structure for analysis. Scandinavian Journal of

Occupational Therapy. (8), 7-18. Recuperado el 6 de abril de 2013 de http://kurs.slu.se/kurser/lp0418/20057.0607/lenakarin20041206.pdf
Richards, H. (2012). Educación para la Integración Social. Revista Interdisciplinaria del Centro de Políticas para el Desarrollo Magíster en Gerencia y Políticas Públicas. 5(2), 4-20. Recuperado el 16 de noviembre de 2013 de http:// www.revistas.usach.cl/ojs/index.php/politicas/article/viewFile/1066/1005\#.

SENAME Servicio Nacional de Menores (2013). Departamento de Protección de Derechos Orientaciones Técnicas Específicas Modalidad CREAD. Recuperado el 25 de octubre de 2013 de http://www.sename.cl/wsename/estructuras.php?name $=\mathrm{C}$ ontent $\&$ pa $=$ showpage $\&$ pid $=14$

Sneiderman S. (2011). Consideraciones acerca de la Confiabilidad y Validez en las Técnicas

Proyectivas. Recuperado el 5 de septiembre de 2013 de http://www. scielo.org.ar/pdf/spc/v15n2/v15n2a05.pdf.

Wilcock, A.A. (2006). An occupational theory of human nature. Chapter 2: An occupational perspective of health (Second Edition). Thorofare, N.J.: Slack Incorporated. 